\title{
Doi Moi en Vietnam
}

\author{
Rosu Ana-Maria
}

Desde el ano 1986, Vietnam se vio involucrado en un proceso de renovación para promover el desarrollo socioeconómico y una integración adecuada con el resto del mundo. Han surgido una serie muy larga de problemas teoréticos y practicos con respecto a la vida de los ciudadanos $\mathrm{y}$, por otra parte, con respecto a las relaciones extranjeras. "El flamante gobierno comunista que terminó por tomar el control del país en 1975 introdujo un modelo político caracterizado por un entramado institucional poco transparente, la planificación central en la toma de decisiones, limitaciones a las iniciativas privadas y controles sobre el sistema productivo. Las intenciones eran las mejores: lograr la justicia en la repartición de la riqueza. Sin embargo, tras más de una década de difícil situación económica y social, en 1986 el gobierno decidió emprender una serie de reformas orientadas a la reducción de la pobreza y a la promoción del crecimiento económico". (Doi Moi: La apertura como motor del desarrollo socioeconomico de Vietnam, Rebeca Vidal)

Estas reformas se conocen como Doi Moi, que denomina renovación o nuevo pensamiento. Antes de Doi Moi, Vietnam era un país con un bajo nivel de desarrollo, fuertemente dañado por la guerra. Tanto su economía como su sociedad estaba dominada por lo tradicional, ya que su populación emprendia actividades con técnicas rudimentarias- la agricultura, la artesanía etc. El Estado había fundado sus políticas de desarrollo basadas en los principios de planificación central y propiedad colectiva. Esto permitió la toma de una decisión activa en la movilización y asignación de los recursos nacionales para lograr objetivos sociales. A pesar su bajo nivel de desarrollo económico, Vietnam fue capaz de realizar de manera justa logros notables en materia de desarrollo humano, especialmente en los ámbitos de la educación y salud.

Sin embargo, a principios del año 1980, las debilidades en el modelo de planificación central comenzaron a manifestarse. La información era inadecuada o distorsionada, los incentivos eran débiles o inexistentes y los recursos eran repartidos de manera ineficiente. El crecimiento económico estancó, la inflación subió rápidamente y las finanzas públicas se vieron afectadas. La gente tenía muy pocas oportunidades para disfrutar de trabajos significativos y ganar ingresos decentes. La situación se agravó por el agotamiento de la ayuda de la Unión Soviética y la crisis socioeconómica dejó claro que el sistema limitaba las iniciativas individuales severamente. Dada esta situacion, el Estado ha implementado algunas medidas orientadas al mercado, reformas, sobre todo a nivel microeconómico.

„Frente a este panorama, el VI Congreso Nacional del Partido Comunista decidió iniciar grandes cambios en la economía, y emprendió acciones tendientes a reducir el protagonismo del Estado en su planificación, permitir una mayor autonomía en el ámbito local; adoptar un modelo de precios y competencia de acuerdo con el mercado a través de la eliminación de controles; ofrecer facilidades para el uso de la tierra y la libertad de cultivos en función del mercado; reconocer la actividad privada como motor del crecimiento económico, y brindar libertad a las empresas estatales y privadas para ejecutar operaciones de comercio exterior." (Doi Moi: La apertura como motor del desarrollo socioeconomico de Vietnam, Rebeca Vidal) 
A consecuencia de este evento resulto una nueva estructura economica y una forma mas eficiente de los recursos humanos, todo esto siendo posible por medio de dar mas autonomia a las empresas, impulsar el desarrollo del sector privado y por la creacion de incentivos para la inversion extranjera y la provision de un mejor acceso a los mercados extranjeros. Durante la etapa Doi Moi se crearon más puestos de trabajo que en el pasado, cuando el Estado y las empresas públicas eran los únicos empleadores y las personas no podían elegir libremente los trabajos.

Las reformas Doi Moi pueden ser partidas en dos etapas, cada una con objetivos diferentes. En la primera etapa se perseguia mejorar la situación dramática en la que estaba Vietnam caracterizada por todo lo que mas arriba esta mencionado. Por lo tanto, el primer set de reformas se trato sobre el sector de la agricultura: descolonización y un cambio hacia una economía basada en la produccion familiar, con el fin de promover la producción nacional de alimentos y limitar las importaciones de dichos bienes. El éxito de las reformas en este sector limitó la brecha de ingresos entre trabajadores rurales y urbanos. Estos resultados contribuyeron a limitar las migraciones durante el período del Doi Moi. Las reformas implementadas se relacionaron con 3 dimensiones: el papel del estado, en el que encontramos, en primer lugar, el viejo sistema de gestión organizado central que dependía de subsidios estatales que ha sido abolido. Tambien se liberalizo el comercio interno y aparece el sector privado, donde las empresas especializadas en la producción de productos básicos son acceptadas por El Partido Comunista y apolladas. Esto fue seguido por la Ley de Empresas Privadas que fue promulgada en 1990 y por la constitución de 1992 que reconocio oficialmente el papel del sector privado. Otra dimensión será la distribución de tierra a los agricultores, que aseguro condiciones optimas para el desarrollo de la producción agrícola, movilizando capital y fuerza de trabajo. Ademas, las rentas vinculadas a la producción efectiva se establecieron por medio de contratos entre los agricultores familiares y sus cooperativas o el Estado, que resulto ser una forma muy eficiente de mejorar la producción a través de la administración que estaba dentro de las familias de agricultores y el fuerte apoyo estatal a los servicios agrícolas.

Tomando en cuenta los buenos resultados obtenidos anteriormente, se creo una segunda serie de reformas que además de la seguridad alimentaria, tenia el objetivo de generar ingresos del comercio exterior y ganar una cuota en el mercado por medio de una estrategia agresiva, basada en precios de exportaciones bajos. Esto tuvo como consecuencia que la agricultura se convirtió, con el tiempo, en la major fuente de ganancias extranjeras. El superávit del sector de agricultura facilito las inversiones en el sector industrial, lo que atrajo fuerza de trabajo del medio rural.

"Estas medidas de liberalización, aunadas a otras específica- mente trazadas para aumentar la productividad, como el aumento de la inversión estatal en sistemas de irrigación, la creación de ban- cos que otorgaran créditos a los pequeños productores y la rápida expansión del acceso a la educación, sistemas de salud y servicios básicos, comenzaron a dar sus frutos más allá de lo estimado originalmente por el gobierno vietnamita." (Vietnam: una potencia comercial enmenos de 25 años- Lecciones de Hanoi)

"Los cambios económicos y sociales continuaron durante la década de los noventa, afirmando las capacidades de una población cada vez mejor educada y deseo sa de trabajar, al tiempo que se profundizaban las diferencias entre lo rural y lo urbano." (Vietnam: una potencia comercial enmenos de 25 años- Lecciones de Hanoi). El mayor desarrollo estuvo en el sector agrícola, que al final tuvo un doble papel: logro aumentar suficientemente como 
para remediar la crisis alimentaria en la que se encontraba Vietnam en el momento de la Renovacion y luego se consolido y se convirtió en un gran exportador con capacidad de contribuir al ingreso del país.

\begin{tabular}{|c|c|c|c|c|c|c|c|}
\hline Human Development Report* & 1995 & 1996 & 1997 & 1998 & 1999 & 2000 & 2001 \\
\hline (Reference year) & (1992) & (1993) & (1994) & (1995) & (1997) & (1998) & (1999) \\
\hline Life expectancy at birth (years) & 65.2 & 65.5 & 66.0 & 66.4 & 67.4 & 67.8 & 67.8 \\
\hline Adult literacy (\%) & 91.9 & 92.5 & 93 & 93.7 & 91.9 & 92.9 & 93.1 \\
\hline Combined enrolment rate (\%) & 49 & 51 & 55 & 55 & 62 & 63 & 67 \\
\hline Real GDP per capita (PPP US\$) & 1,010 & 1,040 & 1,208 & 1,236 & 1,630 & 1,689 & 1860 \\
\hline Human Development Index (value)* & 0.611 & 0.618 & 0.634 & 0.639 & 0.666 & 0.671 & 0.682 \\
\hline Human Developmeft Index (rank)* & 120 & 121 & 121 & 122 & 110 & 108 & 101 \\
\hline
\end{tabular}

Fuente: Nacional Human Development Report 2001, Doi Moi and Human Development in Vietnam

\begin{tabular}{|c|c|c|c|c|c|c|}
\hline \multicolumn{7}{|c|}{$\begin{array}{l}\text { TABLE } 2.3 \\
\text { HDI ranking for selected countries (1999, out of } 162 \text { countries) }\end{array}$} \\
\hline Country & HDI rank & $\begin{array}{l}\text { Life expectancy } \\
\text { (years) }\end{array}$ & $\begin{array}{l}\text { Adult literacy } \\
\text { rate }(\%)\end{array}$ & $\begin{array}{c}\text { Combined } \\
\text { enrolment ratio } \\
(\%)\end{array}$ & $\begin{array}{c}\text { Real GDP } \\
\text { per capita } \\
\text { (PPP\$) }\end{array}$ & $\mathrm{HDI}$ \\
\hline Japan & 9 & 80.8 & 99.0 & 82 & 24,898 & 0.928 \\
\hline Hong Kong & 24 & 79.4 & 93.3 & 63 & 22,909 & 0.880 \\
\hline Singapore & 26 & 77.4 & 92.1 & 75 & 20,767 & 0.876 \\
\hline Malaysia & 56 & 72.2 & 87.0 & 66 & 8,209 & 0.774 \\
\hline Thailand & 66 & 69.9 & 95.3 & 60 & 6,132 & 0.757 \\
\hline Philippines & 70 & 69.0 & 95.1 & 82 & 3.805 & 0.749 \\
\hline China P. R. & 87 & 70.2 & 83.5 & 73 & 3,617 & 0.718 \\
\hline Viet Nam & 101 & 67.8 & 93.1 & 67 & 1,860 & 0.682 \\
\hline Indonesia & 102 & 65.8 & 86.3 & 65 & 2.857 & 0.677 \\
\hline India & 115 & 62.9 & 56.5 & 56 & 2,248 & 0.571 \\
\hline Myanmar & 118 & 56.0 & 84.4 & 55 & 1,027 & 0.551 \\
\hline Cambodia & 121 & 56.4 & 68.2 & 62 & 1,361 & 0.541 \\
\hline Lao PDR & 131 & 53.1 & 47.3 & 58 & 1,471 & 0.476 \\
\hline
\end{tabular}

Fuente: Nacional Human Development Report 2001, Doi Moi and Human Development in Vietnam

\begin{tabular}{lcccc}
\hline $\begin{array}{l}\text { TABLE 2.4 } \\
\text { GDP and Employment by Type of Ownership } \\
\text { (percentages) }\end{array}$ & $\begin{array}{c}\text { GDP by Ownership } \\
\text { Share in 1999 }\end{array}$ & Growth 1996-1999 & Share in 1999 & Growth 1996-1999 \\
\hline & 100.0 & 7.0 & 100.0 & 3.4 \\
\hline Total GDP & 49.4 & 6.9 & 9.0 & 1.5 \\
Public (State \& collective) & 40.5 & 5.1 & 90.4 & 3.4 \\
Non-State (Total) & 33.2 & 4.9 & 89.0 & 3.3 \\
Households \& Farms & 7.3 & 9.7 & 1.4 & 12.1 \\
Private Companies & 10.2 & 18.1 & 0.6 & 38.5
\end{tabular}

Fuente: Nacional Human Development Report 2001, Doi Moi and Human Development in Vietnam 


\section{Bibliografia:}

1. Cima, Ronald J., (1989) Vietnam: a country study, primera edición, Library of Congress Cataloging-in-Publication Data, 1989, Capitulo 4

2. F. Castillo, L. Odell, Marlene, Andrew, (2008) Vietnam in a Nutshell: An Historical, Political and Commercial Overview, International Law Practicum, NYSBA, 2008, Vol. 21, No. 2

3. Vuong, Quan Hoang, (2014) Vietnam's political economy: a discussion on the 1986-2016 period, CEB Working paper no. 14/010, 2014

4. Vidal, Rebeca, (2010) Doi Moi: La apertura como motor socioeconomico de Vietnam, Serie estudios de casos AVE, 2010

5. Perez Saenz, Sandi Meza, Andres, Vinicio, (2007) Vietnam: una potencia comercial en menos de 25 años, Revista Centroamericana de Administración Publica (52-53): 385397,2007

6. Marzin, Michaud, Jacques, Agnalys, (2016) Evolution of Rural Development Strategies and Policies Lessons from Vietnam, October 2016

7. Perez Saenz, Sandi Menza, Andres, Vinicio, (n.d.) Vietnam: una potencia comercial enmenos de 25 años- Lecciones de Hanoi

8. Nacional Human Development Report 2001, (2001) Doi Moi and Human Development in Vietnam, The Political Publishing House, Hanoi, 2001 